\title{
Spline Cúbico para el Tratamiento Funcional de la Radiación Solar Global
}

\author{
Amalia I. Escudero(1), Celso G. Recalde ${ }^{(1,2)}$, Silvia M. Haro(1) y Manuel A. Meneses ${ }^{(2)}$ \\ (1) Escuela Superior Politécnica de Chimborazo, Escuelas de Física Matemática y Ciencias Químicas, \\ Riobamba - Ecuador (e-mail: aisbelescudero@gmail.com, crecalde672000@yahoo.com, \\ silvyharoo@hotmail.com) \\ (2) Universidad Nacional de Chimborazo, Escuela de Ingeniería Ambiental, Riobamba - Ecuador \\ (e-mail: antoniomenesesfreire@hotmail.com)
}

Recibido May. 28, 2015; Aceptado Jul. 9, 2015; Versión final Oct. 2, 2015, Publicado Abr. 2016

\begin{abstract}
Resumen
El artículo muestra el cálculo de los splines cúbicos que definen el comportamiento de la radiación solar global en una localidad ecuatorial alto andina. El suavizado spline se desarrolla en un espacio funcional normado ortogonal donde se transforman los datos discretizados (ti,xi) en funciones $X(t)$. El análisis de datos univariante y multivariante permite estudiar los fenómenos naturales desde conjuntos finitos de valores numéricos. Por otro lado, la recolección de datos implica no solo obtener los valores, sino también involucra los procesos o funciones que les dan origen. Para la detección de datos atípicos se empleó el remuestreo mediante aleatorización muestral (bootstrap), con lo que se obtuvo las medias anuales, mensuales y por períodos climáticos. Los promedios funcionales mensuales de la radiación solar global muestran la presencia de dos formas de comportamiento en el período estudiado.
\end{abstract}

Palabras clave: spline cúbico; análisis de datos funcionales; análisis estadístico; radiación solar global; datos meteorológicos; aleatorización muestral

\section{Cubic Spline for the Functional Treatment of the Global Solar Radiation}

\begin{abstract}
The article shows the calculation of cubic splines that defines the behavior of the global solar radiation on a high Andean equatorial location. The spline smoothing is developed in a normed orthogonal functional space, where the discretized data (ti,xi) are transformed into functions $X(t)$. The analysis of univariate and multivariate data allows studying natural phenomena from finite sets of numerical values. Data collection involves not only getting values, but also includes the processes or functions that give rise to them. Bootstrap resampling was used for detecting outliers with which annual, month and by climatic periods averages are obtained. Functional monthly averages of global solar radiation show the presence of two forms of behavior during the period studied.
\end{abstract}

Keywords: cubic spline; functional data analysis; statistical analysis; global solar radiation; meteorological data; bootstrap 


\section{INTRODUCCION}

En la actualidad la energía solar y otras formas de energías renovables reciben atención de ambientalistas, científicos, economistas, políticos, operadores del servicio eléctrico y muchos otros grupos que promueven el desarrollo sustentable (Yang et al., 2014). La radiación solar incidente da inicio a la cadena energética global, influye en el cambio climático (Serrano et al., 2012), es parámetro primordial para la construcción de modelos y simulaciones en diversos campos de las ciencias e ingenierías para el dimensionamiento de sistemas energéticos (Monné et al., 2011), (Recalde et al., 2015b), estimaciones meteorológicas, efectos globales como la evapotranspiración (Sanchez et al., 2011), entre otros. De la física atmosférica se sabe que el valor de la Radiación Solar Global (RSG) es mayor en sitios más altos, porque al aumentar la altura del lugar la capa atmosférica se hace más delgada, la distancia y la cantidad de materia (aire) que atraviesan los rayos solares disminuye (Igbal, 1983), (Recalde et al., 2015b); a pesar de ello, no es práctico basar las predicciones de RSG únicamente considerando la atenuación de la radiación extraterrestre por la atmósfera; no obstante, en los procesos solares se usan mediciones históricas de radiación solar en la localidad y/o de localidades cercanas similares (Gray et al., 2010) y (Vernich et al., 1996); por otro lado, la información estadística publicada en la literatura profesional es insuficiente para dimensionar y optimizar los sistemas de energía con alta fracción solar, principalmente por la inexistencia de datos en largos periodos de tiempo (Gordon-Reddy, 1988), a pesar de ello existen estudios de radiación solar como: el de Gordón 1988 que presenta un análisis de las propiedades estacionarias y secuenciales de la RSG diaria sobre un plano horizontal en una base mensual discreta, para una serie de lugares de condiciones climáticas diversas por lo que propone una función de densidad de probabilidad sencilla; Bulut 2007 muestra un modelo para estimar la RSG basados en funciones trigonométricas, el cual depende únicamente del día del año; Antonanzas-Torres 2012 hace una comparación de los datos de RSG tomados desde un modelo de estimación satelital y mediciones en la superficie terrestre con técnicas geoestadísticas. El método más usado en investigaciones tanto teóricas como prácticas en el tratamiento de grandes masas de datos es el funcional (Ramsay-Silverman, 1997), donde se considera a $X(X, y, z, t)$ un campo meteorológico (WahbaWendelberger, 1980) que en la práctica es preferible a trabajar con vectores de gran dimensión (FreimanMunís, 2001). Sin embargo, existen pocas publicaciones donde se aplica el análisis de datos funcional en variables meteorológicas; Ocaña en 2009 estimó un modelo funcional de la concentración de polen en cierto intervalo de tiempo desde su comportamiento anterior y de la temperatura; Posada 2015 empleó una técnica multifractal para la zona alto andina que genera campos de lluvia a escala reducida.

Para este trabajo se analizan las variaciones diarias de la RSG del año 2010, en una localidad ecuatorial alto andina como elementos de un espacio de Hilbert; este conjunto de datos funcionales diarios $\left\{X_{1}(t), X_{2}(t), \ldots, X_{365}(t)\right\}$ corresponden a la observación de 365 unidades estadísticas funcionales $X_{1}(t), X_{2}(t), \ldots, X_{365}(t)$ idénticamente distribuidas. El spline cúbico es un método muy usado para la interpolación y ajuste de una curva con derivadas continuas a partir de un conjunto discreto de datos, se empleó la regresión mínimo-cuadrática con base B-splines (Higham, 1992) con secciones de polinomios conectados entre sí (Febrero-Oviedo, 2012) y (Quintero et al., 2010).

En este estudio las funciones atípicas fueron identificadas mediante el remuestreo de Bootstrap (FebreroOviedo, 2012), también se obtuvo la RSG media anual, mensual y por los periodos climáticos definidos por el Instituto Nacional de Meteorología e Hidrología del Ecuador (INAMHI) para la región interandina: período seco de junio a septiembre y período lluvioso de octubre a mayo. Los promedios funcionales mensuales de la RSG mostraron la presencia de dos comportamientos distintos en el intervalo de tiempo estudiado.

\section{METODOLOGÍA}

Se consideró un intervalo de tiempo con datos completos para el análisis de la RSG, utilizando un piranómetro Li-Co \#LI-200SA, con certificado de calibración y error típico del 5\% y un data logger NRG Symphonie instalado en una estación meteorológica a 2480 m.s.n.m, hemisferio sur, zona 17S, a $9816967,05 Y$ y $758398,29 X$. La base de datos corresponde a los valores medios registrados cada 10 minutos en $W / \mathrm{m}^{2}$, formando así una matriz de 52560 datos, equivalentes a 365 filas y 144 columnas igualmente espaciados, obteniéndose 365 datos funcionales de la variabilidad diaria de RSG en la zona de estudio. Se realizó un análisis exploratorio funcional de la RSG mediante el software libre R versión 3.1.2, empleando la librería fda.usc de la Universidad Santiago de Compostela, se migró la información a una base de datos legible para $\mathrm{R}$ mediante una conversión a formato .txt con el software Symphonie Data Retriever.

La librería fda.usc de $\mathrm{R}$ con la herramienta fdata trasforma las observaciones $\left(t_{k}, X_{i}\right)$ en funciones $X_{i}(t)$, donde $t_{k}$ es el tiempo y $X\left(t_{k}\right)$ es la RSG con $\mathrm{k}=0,1,2, \ldots, 144$; en un espacio vectorial $L^{2}$. Se calculó la media funcional mediante la ecuación: 
$\bar{X}(t)=\frac{1}{N} \sum_{i=1}^{N} X_{i}(t)$

Donde $N$ es el número de funciones (365) y $X_{i}(t)$ las funciones.

Los datos atípicos se detectaron con la herramienta outliers.depth.trim método de arranque o también llamado remuestreo de Bootstrap, tomando para el estudio $n b=1000$ remuestras $X_{1}{ }^{*}(t), X_{2}{ }^{*}(t), X_{3}{ }^{*}(t), \ldots, X_{1000}{ }^{*}(t)$ mediante $X_{i}^{*}(t)=X_{i}(t)+Z(t)$ en donde $Z(t)$ tiene una distribución normal con media 0 y covarianza la matriz $y \Sigma_{x}$, donde $\sum_{x}$ es la matriz de covarianza de $X_{1}(t), X_{2}(t), X_{3}(t), \ldots, X_{365}(t)$ y $\gamma$ es parámetro de suavizado (profundidad) que puede ser cualquier estadístico descriptivo, que para este caso se usó la mediana al 95\% de confiabilidad (Febrero-Oviedo, 2012). Del mismo modo se determinó la banda de confianza con el comando fdata.bootstrap() que es un conjunto de curvas que siguen la misma distribución que los datos originales cuya distancia funcional $d\left(T, T^{*}\right)$ es inferior al nivel de significancia $\propto$, donde:

$$
T=T\left(X_{1}(t), X_{2}(t), X_{3}(t), \ldots, X_{365}(t)\right)
$$

$T^{* j}=X_{1}^{* j}(t), X_{2}^{* j}(t), X_{3}^{* j}(t), \ldots, X_{1000^{* j}}(t)$

Como indicador $j$ para muestrear, en éste estudio se tomó $n b=1000$ remuestras de Bootstrap (FebreroOviedo, 2012) usando la media como parámetro de suavizado y el cuantil 95 como ancho de banda. Para medir la distancia $d\left(T, T^{* j}\right)$ se usó una métrica de $L^{2}$ dada por:

$\|X(t)\|_{2}=\left(\int_{t_{\min }}^{t_{\max }} X(t)^{2} d t\right)^{1 / 2}$

Para la interpolación de datos irregulares se determinó $X(t)$ mediante splines cúbicos, (Vazquez R., Solano o., 2013). Para hallar numéricamente los splines cúbicos se tomaron 145 puntos donde $t_{0}<t_{1}<t_{2}<\ldots<t_{144}$ que corresponden a las 24 horas del día, y se interpolaron las funciones para cada intervalo de tiempo $\left[t_{0}, t_{1}\right]$, $\left[t_{1}, t_{2}\right], \ldots,\left[t_{143}, t_{144}\right]$. Se define un nuevo polinomio cúbico $X_{i}$ que representa a $X(t)$ en el intervalo $\left[t_{0}, t_{144}\right]$; es decir:

$X(t)=\left\{\begin{array}{rrl}x_{0}(t) & & t \in\left[t_{0}, t_{1}\right] \\ x_{1}(t) & & t \in\left[t_{1}, t_{2}\right] \\ & \cdot & \\ & \cdot & \\ x_{144}(t) & & t \in\left[t_{143}, t_{144}\right]\end{array}\right.$

Se verificó que los polinomios cumplan las siguientes condiciones:

a) Los valores de la función de polinomios adyacentes deben ser iguales en los tiempos interiores o nodos.

b) La primera y última función deben pasar a través de los tiempos extremos.

c) Los polinomios $X_{k}(t): k=0,1,2, \ldots, 144$ tiene la misma pendiente y concavidad en los nodos que las unen,

$X_{k-1}^{\prime}\left(t_{k}\right)=X_{k}^{\prime}\left(t_{k}\right)$

$X^{\prime \prime}{ }_{k-1}\left(t_{k}\right)=X_{k}{ }_{k}\left(t_{k}\right)$

Las ec. 6 y 7 aseguran que $X(t)$ tiene primera y segunda derivadas continuas en $\left[t_{0}, t_{144}\right]$ como elementos de $L^{2}$. Ya que cada par de nodos está unido a un polinomio cúbico, la segunda derivada dentro de cada intervalo es lineal. A ésta se la puede representar mediante un polinomio de interpolación de Lagrange de primer grado ec. 8, esto es:

$x^{\prime \prime}(t)=x^{\prime \prime}\left(t_{k}\right) \frac{t-t_{k+1}}{t_{k}-t_{k+1}}+x^{\prime \prime}\left(t_{k+1}\right) \frac{t-t_{k}}{t_{k+1}-t_{k}}$ 
Denotamos con: $h_{k}=t_{k+1}-t_{k}, y \quad \sigma_{k}=X^{\prime \prime}\left(t_{k}\right)$; por lo que remplazando en la ec. 8 tenemos:

$x^{\prime \prime}(t)=\frac{\sigma_{k}}{h_{k}}\left(t_{k}-t\right)+\frac{\sigma_{k+1}}{h_{k}}\left(t-t_{k}\right)$

Donde $h_{k}$ y $\sigma_{k}$ son constantes, correspondiendo en este caso $h_{k}=10 \mathrm{~min}$. Integrando 2 veces la ec. 9 se tiene:

$X_{k}(t)=\frac{\sigma_{k}}{6}\left[\frac{\left(t_{k+1}-t\right)^{3}}{h_{k}}-h_{k}\left(t_{k+1}-t\right)\right]+\frac{\sigma_{k+1}}{6}\left[\frac{\left(t-t_{k}\right)^{3}}{h_{k}}-h_{k}\left(t-t_{k}\right)\right]+y_{k}\left[\frac{\left(t_{k+1}-t\right)}{h_{k}}\right]+y_{k+1}\left[\frac{\left(t-t_{k}\right)}{h_{k}}\right]$

con $k=0,1,2, \ldots, 144 ;$ que es la ecuación del spline $X(t)$.

Para ilustrar lo anterior se tomaron los datos de RSG de 6:30 a 7:00 de la mañana del primer día del año estudiado.

\begin{tabular}{|l|l|l|l|l|}
\hline$t_{k}$ & $t_{38}=6: 30$ & $t_{39}=6: 40$ & $t_{40}=6: 50$ & $t_{41}=7: 00$ \\
\hline$R(t)\left(W / m^{2}\right)$ & 2,3 & 3,5 & 11,3 & 36 \\
\hline
\end{tabular}

$h_{38}=6: 40-6: 30=0: 10$

$h_{39}=6: 50-6: 40=0: 10$

$h_{40}=7: 00-6: 50=0: 10$

Se determinaron los coeficientes $\sigma_{38}, \sigma_{39}, \sigma_{40}, \sigma_{41}$ con:

$h_{k-1} \sigma_{k-1}+2\left(h_{k-1}+h_{k}\right) \sigma_{k}+h_{k} \sigma_{k+1}=6\left[\frac{y_{k+1}-y_{k}}{h_{k}}-\frac{y_{k}-y_{k-1}}{h_{k-1}}\right]$

y $k=38,39,40,41$ formamos un sistema de orden 3 .

Considerando $\sigma_{38}=X^{\prime \prime}\left(t_{38}\right)$ y $\sigma_{41}=X^{\prime \prime}\left(t_{41}\right)$ entonces $\sigma_{38}=0$ y $\sigma_{41}=0$. Reemplazando los datos en la ec. 11 se tiene:

Para $k=39$

$0,1 \sigma_{38}+2(0,1+0,1) \sigma_{39}+0,1 \sigma_{40}=6\left[\frac{1,3-3,5}{0,1}-\frac{3,5-2,3}{0,1}\right]$

$0,1 \sigma_{38}+0,4 \sigma_{39}+0,1 \sigma_{40}=396$

Para $k=40$

$0,1 \sigma_{39}+2(0,1+0,1) \sigma_{40}+0,1 \sigma_{41}=6\left[\frac{\beta 6-11,3}{0,1}-\frac{11,3-3,5}{0,1}\right]$

$0,1 \sigma_{39}+0,4 \sigma_{40}+0,1 \sigma_{41}=1014$

Con las ec. 12 y 13 obtenemos el sistema:

$\left\{0,1 \sigma_{38}+0,4 \sigma_{39}+0,1 \sigma_{40}=396\right.$

$\left\{0,1 \sigma_{39}+0,4 \sigma_{40}+0,1 \sigma_{41}=1014\right.$

Resolviendo la ec. 14 se tiene: $\sigma_{39}=380$ y $\sigma_{40}=2440$; por lo que los splines en cada intervalo serán:

$X_{38}(t)=633,33(t-6,5)^{3}+28,67(t-6,3)+23(6,4-t)$

$x_{39}(t)=633,33(6,5-t)^{3}+28,67(6,5-t)+72,33(t-6,4)+4066,67(t-6,4)^{3}$

$X_{40}(t)=4066,67(7-t)^{3}+72,33(7-t)+360(t-6,5)$

Se obtiene la segunda función correspondiente al día 2 dada por:

$X_{38}^{\prime}(t)=1353,33(t-6,3)^{3}+54,47(t-6,3)+24(6,4-t)$ 
Una vez obtenidos los splines cúbicos $X(t)$ en cada intervalo $\left[t_{i}, t_{i+1}\right]$, se halla la norma ec. 4.

$\|X\|_{2}=\sqrt{\int_{6,3}^{6,4}\left[720(t-6,3)^{3}+25,8(t-6,3)+(6,4-t)\right]^{2} d t}$

$\|X\|_{2}=8,24 \mathrm{~W} / \mathrm{m}^{2}$

Del mismo modo se procede para todos los intervalos de las funciones.

\section{RESULTADOS Y DISCUSIÓN}

Se identificó las funciones diarias atípicas y se halló las medias funcionales: anual, mensual y por períodos climáticos. La (Fig.1) muestra las 4 funciones atípicas detectadas mediante el remuestreo de Bootstrap, que corresponden aproximadamente al $1 \%$ del total. Los días identificados como atípicos fueron: el 14 de febrero, 26 de febrero, 8 de abril y 20 de octubre, estos presentan cambios bruscos de los valores de la RSG y valores por debajo de la media general, superando así la distancia $d\left(T, T^{*}\right)$ al $95 \%$ de confiabilidad.

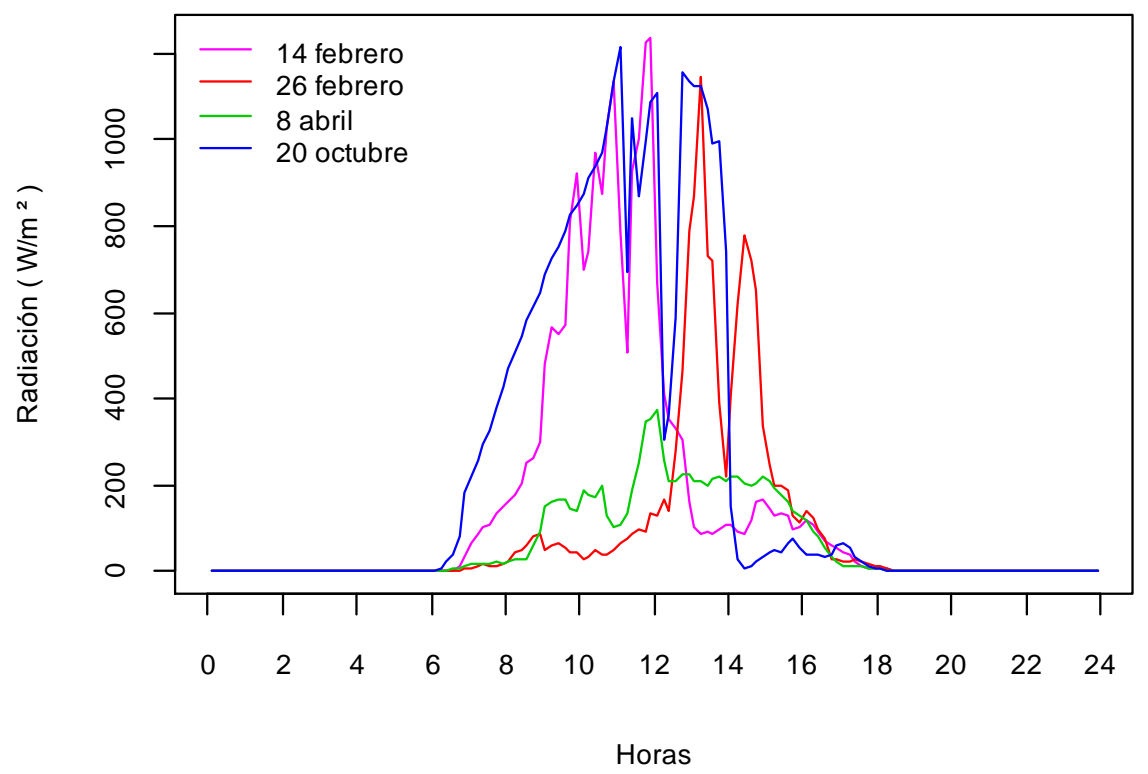

Fig. 1: Funciones atípicas

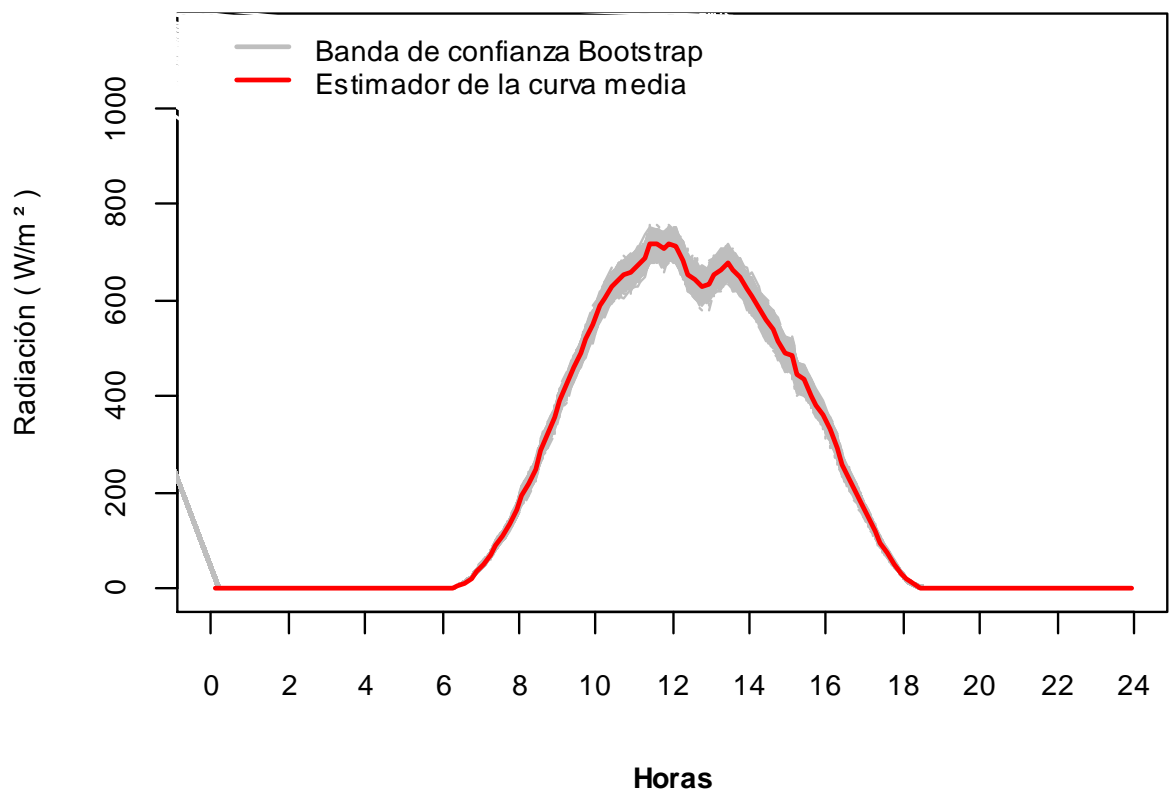

Fig. 2: Media funcional de radiación solar global 2010 
La (Fig. 2) muestra la media funcional de la RSG y su banda de confianza al 95\%, durante el año de estudio, el dataloger registró la RSG a partir de las 06:00 hasta las 18:50 y alcanzó un máximo de 718 $W / m^{2}$. Las horas con mayor radiación solar comprenden desde las 11:30 a 12:10 que superan los 700 $\mathrm{W} / \mathrm{m}^{2}$, además, se puede notar una concavidad en su comportamiento desde aproximadamente: 12:20 a 13:10.

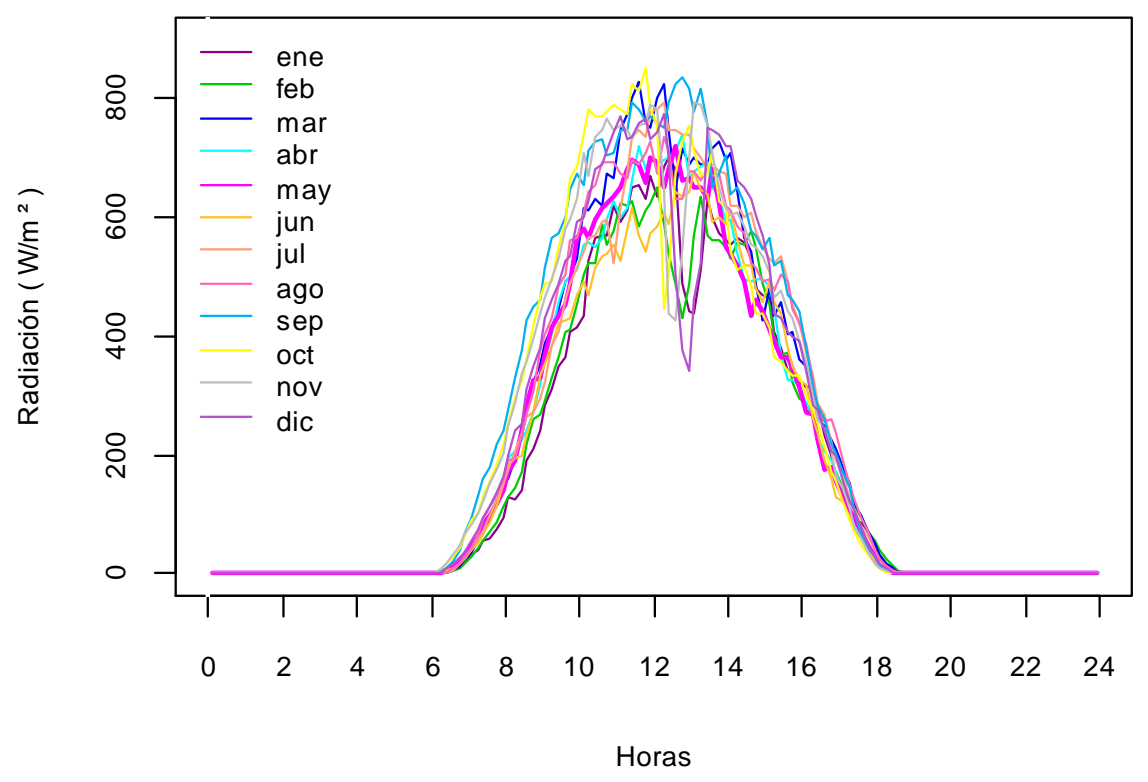

Fig. 3: Radiación solar media mensual

Se construyeron las curvas medias mensuales del comportamiento de la RSG (Fig. 3) las cuales mostraron en general una forma acampanada, sin embargo, los meses de: enero, febrero, octubre, noviembre y diciembre presentan concavidad; por otro lado, marzo, septiembre y octubre en que la Tierra está sobre los equinoccios presentan los mayores valores de la RSG, similar a lo obtenido por (Álvarez et al., 2014) en los Andes a $4^{\circ}$ Sur, a (Olatomiwa et al., 2015) en Nigeria a una latitud de $8^{\circ}$ Norte donde los mayores valores de RSG fueron en los meses de marzo y febrero y (Umoh et al., 2014) a $5^{\circ}$ de latitud Norte obtuvo que los mayores valores de RSG se presentaron en los meses de octubre, diciembre y marzo. Se analizó por separado el período lluvioso que corresponde a: enero, febrero, marzo, abril, mayo, octubre, noviembre y diciembre (Fig. 4) y el período seco que corresponde a junio, julio, agosto y septiembre (Fig. 5) (Recalde et al., 2015a) para determinar características propias.

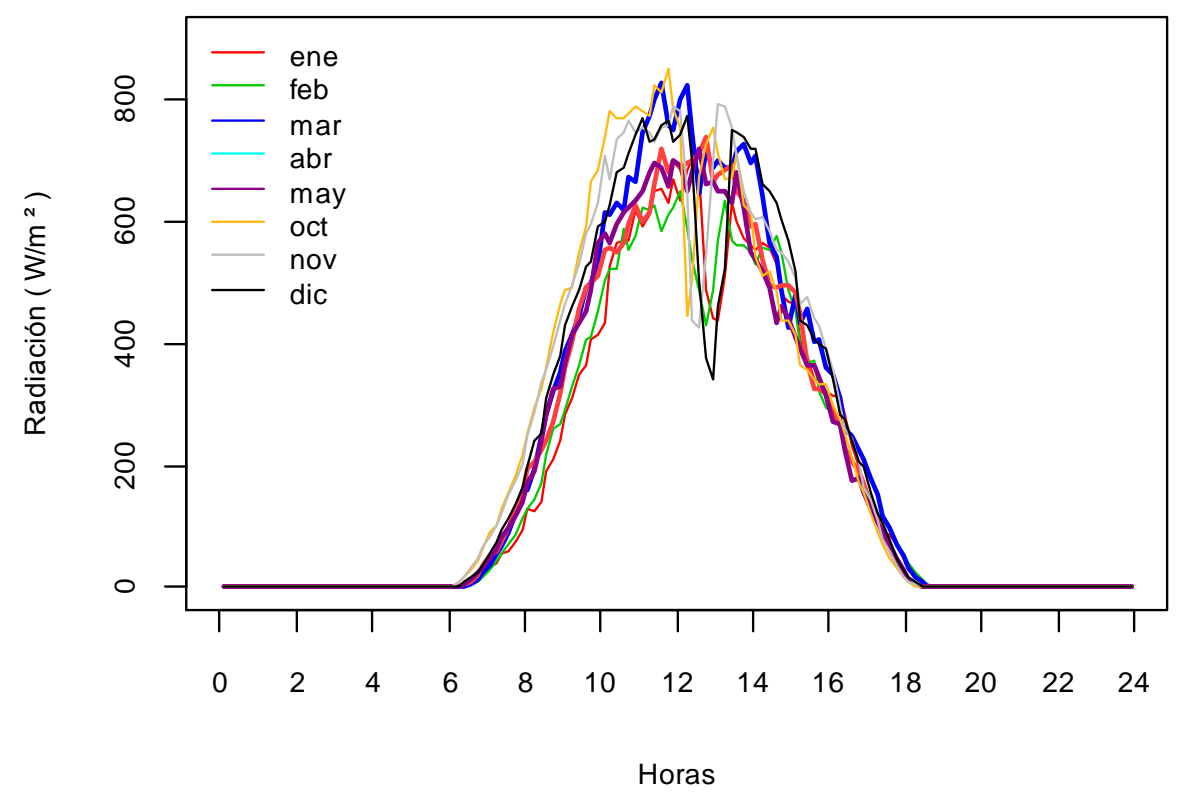

Fig. 4: Radiación solar global media período lluvioso 


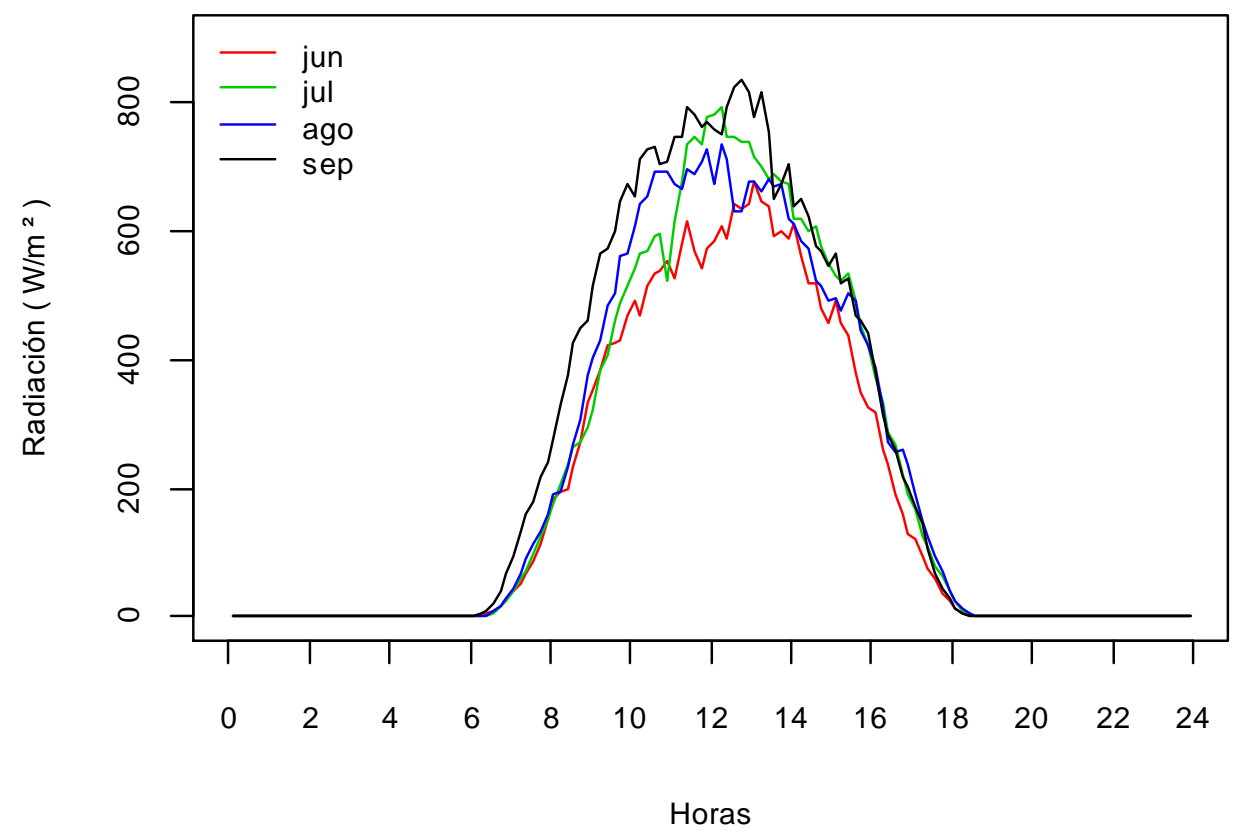

Fig. 5: Radiación solar global media mensual período seco

Para el período lluvioso los meses con media funcional de RSG más elevada (Fig.4) fueron: marzo, octubre, noviembre y diciembre con valores que alcanzan hasta los $824 \mathrm{~W} / \mathrm{m}^{2}$, el mes de marzo presentó una concavidad menor posiblemente por el cambio de período de lluvioso a seco; abril y mayo presentan un comportamiento similar a los meses del período seco (Fig. 5) sin concavidad. Para el período seco los meses con media funcional de RSG (Fig. 5) más elevados fueron julio y septiembre con valores que alcanzan hasta los $838 \mathrm{~W} / \mathrm{m}^{2}$; la diferencia de los valores más elevados de la RSG medida entre periodos lluvioso y seco no fue superior a $24 \mathrm{~W} / \mathrm{m}^{2}$, dado que en la zona ecuatorial andina las estaciones no son claramente marcadas; no obstante, los resultados obtenidos por (Nwankwo et al., 2015), a latitud $6^{\circ}$ Norte y $58 \mathrm{msnm}$, la diferencia de la RSG entre periodo lluvioso y seco fue superior a $400 \mathrm{~W} / \mathrm{m}^{2}$.

La (Fig. 6) muestra comportamiento suave de la RSG en las horas de la mañana aproximadamente hasta las 10:00 y en la tarde a partir de las 16:00, debido a que en estos intervalos predomina la radiación solar difusa (Recalde et al., 2015a).

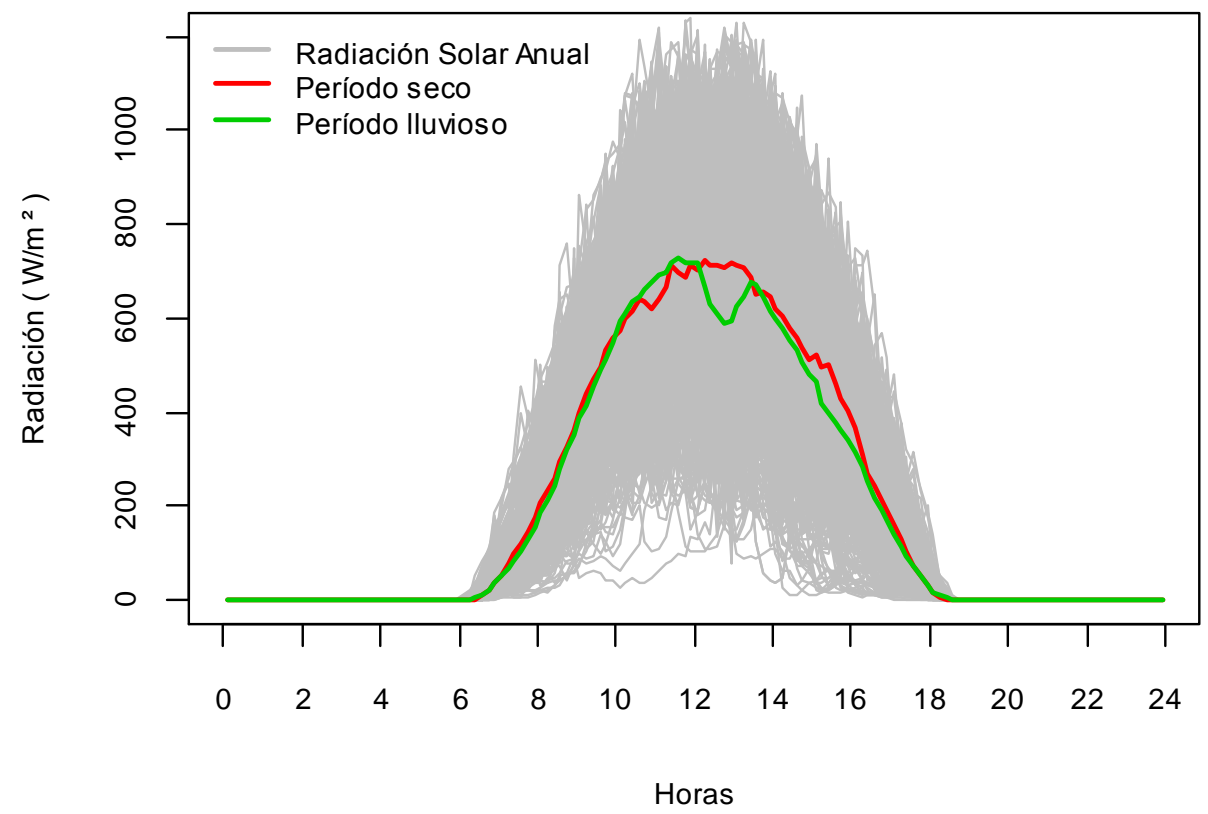

Fig. 6: Radiación solar global media en el período lluvioso y seco 


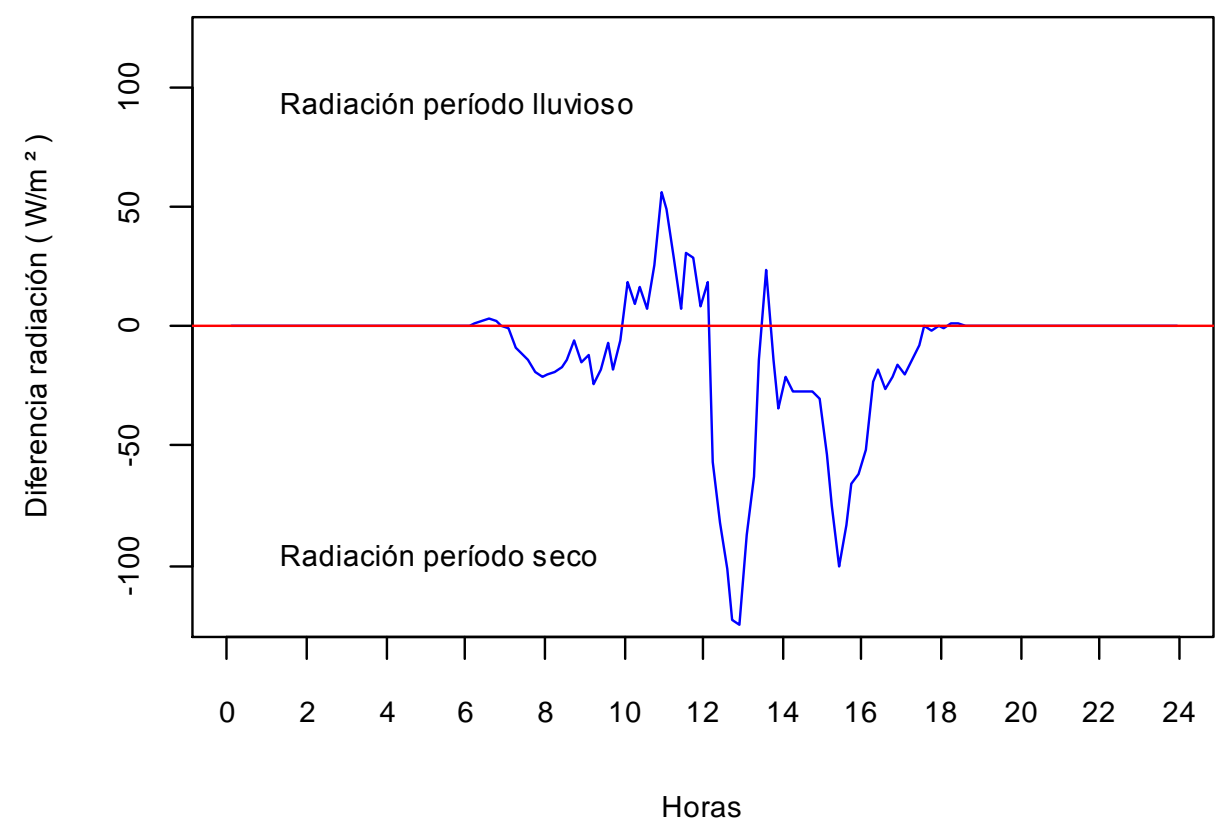

Fig. 7: Comparación media funcional en el período lluvioso y seco

Finalmente al comparar las curvas medias diarias de RSG (Fig. 7) en ambos períodos, se logra identificar que el $65 \%$ de éstas son mayores en el período seco, resultado similar al obtenido por (Dos Santos et al., 2014) en Brasil a una latitud de aproximadamente $9^{\circ}$ Sur y (Nwankwo et al., 2015).

\section{CONCLUSIONES}

La caracterización visual de los datos funcionales sin previos resúmenes facilita la comprensión del comportamiento de la RSG a través del tiempo, que para el caso de la zona ecuatorial andina sin cambios importantes entre períodos climáticos el tratamiento funcional diario describe de mejor manera la RSG.

Los promedios funcionales mensuales de la RSG muestran la presencia de dos comportamientos distintos en el período estudiado, sin embargo, no coinciden con los períodos climáticos determinados por INAMHIEcuador para la región interandina.

\section{REFERENCIAS}

Álvarez, O., Montaño, T., Maldonado, J., La radiación solar global en la provincia de Loja, evaluación preliminar utilizando el método de Hottel, Ingenius, ISSN: 1390-650X, 11, 25-31 (2014)

Antonanzas, F., Sodupe, E., Sanz, A., Fernandez, R., Martinez, F. Technical feasibility assessment of integrated solar combined cycle power plants in Ciudad Real (Spain) and Las Vegas (USA), doi: 10.1007/978-3-319-01854-6, In Proc. 16th International Congress on Project Engineering, (en línea), 18 (2), 1282-1291 (2012)

Bulut, H., Büyükalaca, O., Simple model for the generation of daily global solar-radiation data in Turkey, doi:10.1016/j.apenergy.2006.10.003, Applied Energy, (en línea), 84(5), 477-491 (2007)

Dos Santos, C., De Souza, J., Junior, R., Tiba, y otros seis autores más, On modeling global solar irradiation using air temperature for Alagoas State, doi:10.1016/j.energy.2014.04.116, Northeastern Brazil Energy, (en línea), 71, 388-398 (2014)

Febrero, B., Oviedo, M., Statistical computing in functional data analysis: The R package fda.usc, Journal of Statistical Software, ISSN 1548-7660, (en línea), 51(4), 1-28, 2012. https://www.researchgate.net/profile/Manuel_Oviedo_De_La_Fuente/publication/260552565_Statistical_Co mputing_in_Functional_Data_Analysis_The_R_Package_fda.usc/links/02e7e53189d39c2a6e000000.pdf. Acceso: 5 de Mayo (2014)

Freiman, R., Munís, G., Trimemed means for functional data, doi: 10.1007/BF02595706; Sociedad de Estadística e Investigación Operativa, (en línea), 10(2), 7-13 (2001) 
Gordon, J., Reddy, T., Time series analysis of daily horizontal solar radiation, doi:10.1016/0038092X(88)90139-9, Elsevier, (en línea), 215-226 (1988)

Gray, L. y otros doce autores, Solar influences on climate, doi: 10.1029/2009RG000282, Rev. Geophys, (en línea), 1-53 (2010)

Higham, D., Monotonic piecewise cubic interpolation, with applications to ODE Plotting, doi:10.1016/03770427(92)90205-C, Computational and Applied Mathematics, (en línea), 287-294 (1992)

Igbal, M., An Introduction to solar radiation,1a edición, 303-332, Academic Press Inc, ISBN: 0-12-373750-8, Nueva York, (en línea), (1983)

Monné C., Alonso S., Palacin F., Evaluación de una instalación de refrigeración por absorción con energía solar, Inf. Tecnol. ISSN 0718-0764, (en línea), 22(3), 39-44, 2011. http://www.scielo.cl/scielo.php?script=sci_arttext\&pid=S0718-07642011000300006. Acceso: 10 enero (2014)

Nwankwo S., Nnabuchi, M., Global solar radiation measurement in AbakalikiEbonyi State Nigeria using locally made pyranometer, International Journal of Research, e-ISSN: 2348-6848, (en línea), 2(4), 566-573, 2015. http://internationaljournalofresearch.org/index.php/ijr/article/download/1825/1717. Acceso: 22 de junio (2015)

Ocaña, F., Valderrama, J., Ocaña, F., Escabias, M., Functional modelling in environmetrics, doi: 10.1002env.696, In the XIII international conference on applied stochastic models and data analysis, (en línea), 194-198 (2009)

Olatomiwa, L., Mekhilef, S., Shamshirband, S., Petkovic, D., Potential of support vector regression for solar radiation prediction in Nigeria, doi: 10.1007/s11069-015-1641-x, Natural Hazards, (en línea), 77(2), 10551068 (2015)

Posadas A., Duffaut L., Yarlequé C., Carbajal M., Heidinger H., Carvalho L., Quiroz R., Spatial random downscaling of rainfall signals in Andean heterogeneous terrain, doi:10.5194/npg-22-383-2015, Nonlin. Processes Geophys, (en línea), 383-402 (2015)

Quintero E., Ureña W., Gallego H., Interfaz gráfica para la interpolación de datos a través de splines cúbicos, Scientia Et Technica, ISSN: 0122-1701, (en línea), 16(44), 195-200, 2010. http://www.redalyc.org/articulo.oa?id=84917316035. Acceso: 1 de febrero (2015)

Ramsay, J., Silverman, W., Functional Data Analysis, 1-2. Springer, ISBN: 0-387-95414-7, New York (1997)

Recalde, C., Cisneros, C., Ávila, C., Urquizo, G., Influencia del ángulo de inclinación de los tubos solares evacuados sobre la temperatura del agua, doi: 10.4067/S0718-07642015000400012, Inf. Tecnol. (en línea), 26(4), 89-96 (2015a)

Recalde, C., Cisneros, C., Vaca, D., Ramos, C., Relación de la transmitancia atmosférica con la eleofanía y la diferencia de temperaturas extremas diarias en la zona ecuatorial Andina, doi: 10.4067/S071807642015000100016, Inf. Tecnol. (en línea), 26(1), 143-150 (2015b)

Sanchéz M., Carvacho L., Comparación de ecuaciones empíricas para el cálculo de la evapotranspiración de referencia en la Región del Libertador General Bernardo O'Higgins, Geografía Norte Grande, ISSN 07183402, (en línea), 50, 171-186, 2011. http://www.scielo.cl/scielo.php?pid=S071834022011000300010\&script=sci_arttext. Acceso: 12 de enero (2014)

Serrano S., Zuleta D., Moscoso V., Jácome P., Palacios E., Villacis M., Análisis estadístico de datos meteorológicos mensuales y diarios para la determinación de variabilidad climática y cambio climático en el Distrito Metropolitano de Quito, La Granja, ISSN: 1390-3799, (en línea), 23-47, 2012. http://lagranja.ups.edu.ec/documents/1317427/2990285/Serrano_Meteorologico.pdf. Acceso: 14 de enero (2014)

Umoh, M., Udo, S., Udoakah, Y., Estimating global solar radiation on horizontal surface from sunshine hours over Port Harcourt, doi: 10.5897/JEEER2013.0469, Journal of Electrical and Electronics Engineering Research, (en línea), 6(1), 1-5 (2014)

Vazquez R., Solano O., Modelación espacial de la lluvia y la evapotranspiración teniendo en cuanta parámetros geográficos, Ciencias de la Tierra y el Espacio, ISSN 1729-3790, (en línea), 14(1), 80- 90, 2013. 
http://www.iga.cu/publicaciones/revista/assets/08.interpolacio.-agroclimaticaok27.2.13.pdf. Acceso: 10 de Noviembre (2013)

Vernich, L., Zuanni, F., About the minimun number of years required to stabilize the solar irradiation stadistical estimates, doi: http://dx.doi.org/10.1016/S0038-092X(96)00121-1, Solar energy, (en línea), 57(6), 445-447 (1996)

Wahba G., Wendelberger J., Some new Mathematical methods for variational objetive analysis using splines and cross validations, doi: http://dx.doi.org/10.1175/1520-0493(1980)108<1122:SNMMFV>2.0.CO;2, American Meteorological Society, (en línea), 108, 1122-1143 (1980)

Yang, D., Walsh, W., Jirutitijaroen, P., Estimation an aplication of clear sky global horizontal irradiance at the Equator, doi: 10.1115/1.4027263, Solar Energy Engineering, (en línea), 136(3), 1-4 (2014) 